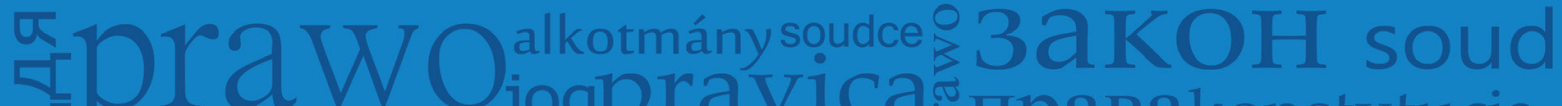

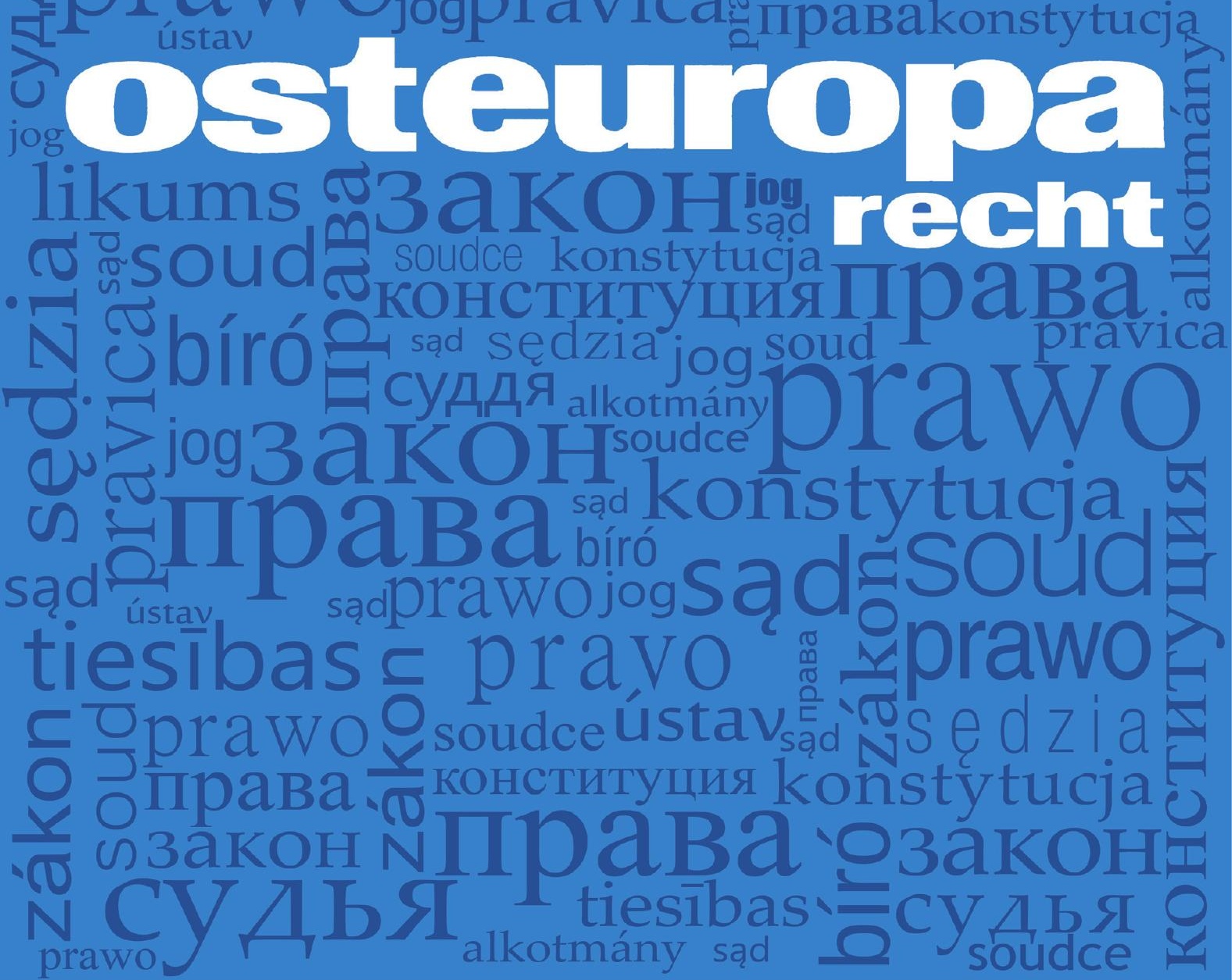

\title{
Rechtsharmonisierung
} in der östlichen Partnerschaft

Georgien und Ukraine

Unternehmensstrafrecht

Russland

Umweltrecht

Ungarn

\section{prawoзаконјog 4/2016}

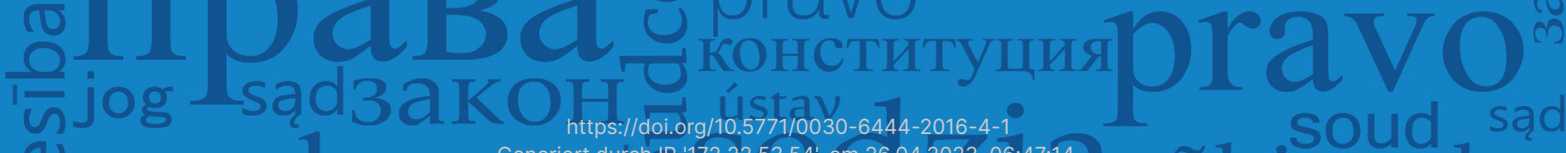


Im Auftrag der

\section{DEUTSCHEN GESELLSCHAFT FÜR OSTEUROPAKUNDE e. V. C}

herausgegeben von Prof. Dr. Burkhard Breig, Berlin, Prof. Dr. Caroline von Gall, Köln, Richterin am Europäischen Gerichtshof für Menschenrechte Prof. Dr. Dr. h.c. Angelika Nußberger M. A., Straßburg/Köln, Dr. Carmen Schmidt, Köln, Prof. DDr. Bernd Wieser, Graz, Prof. Dr. Dr. h.c. (Ternopil) Fryderyk Zoll, Osnabrück und Krakau

unter Mitwirkung von Prof. Dr. habil. Bogustaw Banaszak, Breslau, Prof. Dr. F. J. M. Feldbrugge, Leiden, Prof. Dr. Dr. h.c. Martin Fincke, Passau, Prof. Dr. Anders Fogelklou, Uppsala, Prof. Dr. Michael Geistlinger, Salzburg, Verfassungsrichter Prof. Dr. Pavel Holländer, Brünn, Prof. Dr. Peeter Järvelaid, Tallinn, Richter am Gerichtshof der Europäischen Union Dr. Egil Levits, Luxemburg, Prof. Dr. Otto Luchterhandt, Hamburg, Botschafter a. D. Dr. Klaus Schrameyer, Bonn, Prof. Dr. Dres. h.c. FriedrichChristian Schroeder, Regensburg, Prof. Dr. Theodor Schweisfurth, Heidelberg, Prof. Dr. Dr. h.c. László Sólyom, Staatspräsident a.D., Präsident des Verfassungsgerichts a.D., Budapest, Prof. Dr. Alexander Trunk, Kiel

\section{REDAKTION:}

Prof. Dr. Burkhard Breig, Prof. Dr. Caroline von Gall, Munir Qureshi (Dipl.-Übers.), Dr. Carmen Schmidt, Prof. DDr. Bernd Wieser, Prof. Dr. Dr. h.c. (Ternopil) Fryderyk Zoll

Anschrift:

Dr. Carmen Schmidt

Institut für osteuropäisches Recht und Rechtsvergleichung

Klosterstr. 79d

50931 Köln

E-Mail: oe-recht@dgo-online.org, Aus dem Schrifttum: bernd.wieser@uni-graz.at, Aus Justiz und Rechtsprechung: cvgall@uni-koeln.de, Aus der Forschung: burkhard.breig@fu-berlin.de

Die in OSTEUROPA RECHT veröffentlichten Beiträge mit Verfasserangabe geben die Ansicht der betreffenden Autoren wieder, nicht die der Herausgeber oder der Redaktion.

\section{OSTEUROPA RECHT}

Erscheinungsweise: dreimonatlich. Bezugsmöglichkeiten: Bestellungen nehmen der Verlag und der Buchhandel entgegen. Bezugszeit: Das Abonnement gilt zunächst für ein Jahr; es verlängert sich, wenn nicht drei Monate vor Ablauf dieses Jahres schriftlich gekündigt wird. Darüber hinaus sind Kündigungen jeweils drei Monate vor Jahresende der Abonnementverwaltung mitzuteilen. Bezugspreise: Einzelheft 22,- $€$. Jahresabonnement Printausgabe 76,- $€$ (für Mitglieder der Dt. Gesellschaft für Osteuropakunde e. V. sowie für Studierende (gg. Nachweis) 57,- $€$ ). Jahresabonnement Kombiversion (Print + digital) 98,- $€$ (für Mitglieder der Dt. Gesellschaft für Osteuropakunde e. V. sowie für Studierende (gg. Nachweis) 74,- $€$ ). Alle Preise für ein Jahresabonnement inkl. MwSt., zzgl. Versandkosten (9,- $€$ Inland/17,80€ Ausland) bei Printausgaben. Preise der Print- und Onlineausgabe für Institutionen mit IP-Zugang auf Nachfrage beim Verlag.

Verlag und Abonnementverwaltung: BWV • Berliner Wissenschafts-Verlag GmbH, Markgrafenstraße 12-14, 10969 Berlin, Telefon (030) 8417 70-0, Telefax (030) 84 17 70-21,

E-Mail: bwv@bwv-verlag.de

Gedruckt auf alterungsbeständigem Papier

Die in dieser Zeitschrift veröffentlichten Beiträge sind urheberrechtlich geschützt. Für den Fall der Annahme und Veröffentlichung eines eingereichten Manuskripts geht das räumlich unbeschränkte, ausschließliche Werknutzungsrecht für die Dauer des gesetzlichen Urheberrechts vom Autor/von den Autoren an den Verlag über. 


\section{osteuropa recht}

Fragen zur Rechtsentwicklung in Mittel- und Osteuropa sowie den GUS-Staaten 62. Jahrgang, Heft 4|2016 „Rechtsharmonisierung in der östlichen Partnerschaft““

\section{INHALT DIESES HEFTS}

EDITORIAL

AUFSÄTZE

Dodo Shengelia

Die Angleichung des georgischen Urheberrechts an EU-Recht - Software- und Umgehungsschutz

Volodymyr Kochyn

Tímea Drinóczi

Sergij Rabinovych/

Oleg Pankevych

Michael Denga

László Fodor
European Economic Interest Grouping (EEIG): Implementation in Ukraine

Special Legal Orders: Challenges and Solutions

Der Gleichheitssatz in der Ukraine

S. 412

Entwicklungen des „Betrugs im Bereich unternehmerischer Tätigkeit“" in Russland

Kommunales Umweltrecht in Ungarn

\section{AUS DEM SCHRIFTTUM}

Claudia Schubert/Alexander M. Kurennoy (Hrsg.), Handbuch für deutsches und russisches Arbeitsrecht (Dmitry Marenkov), S. 484 
UNGARN: Verfassungsgerichtsurteil 12/2016. (VI. 22.) AB über die Volksabstimmung gegen die „Ansiedlung von Ausländern durch die EU“, S. 487; Verfassungsgerichtsurteil 3153/2016. (VII. 22.) AB über die Reichweite des Rechts auf gerichtlichen Rechtsschutz, S. 488; Verfassungsgerichtsurteil 13/2016. (VII. 18.) AB über gesetzgeberisches Unterlassen bei einer Grundrechtskollision, S. 488 (Herbert Küpper)

\section{AUS DER RECHTSPRECHUNG DES EGMR}

UNGARN: Freie Rede im Parlament, S. 490; Baka zum Zweiten: die vorzeitige Entlassung des Präsidenten des Obersten Gerichts, S. 491; Magyar Keresztény Mennonita Egyház et al. zum Zweiten: Entschädigung, S. 492; Schematische Anordnung von spezieller Flüchtlingshaft für Flüchtlinge, S. 492 (Herbert Küpper)

ABSTRACTS

AUTOREN

S. 496 